\title{
Parallel Multilevel Preconditioners
}

\author{
James H. Bramble, Joseph E. Pasciak, and Jinchao Xu
}

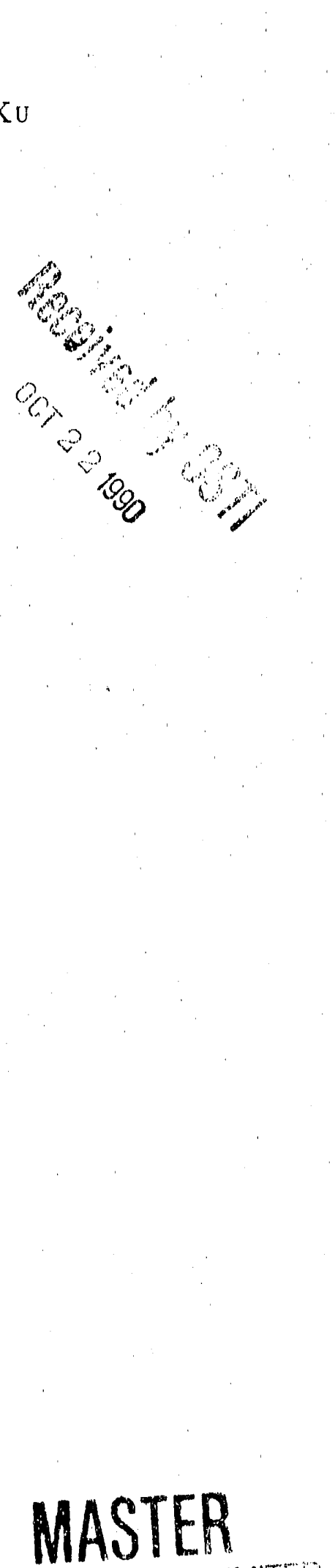

\section{DISCLAIMER}

This report was prepared as an account of work sponsored by an agency of the United States Government. Neither the United States Government nor any agency thereof, nor any of their employees, makes any warranty, express or implied, or assumes any legal liability or responsibility for the accuracy, completeness, or usefulness of any information, apparatus, product, or process disclosed, or represents that its use would not infringe privately owned rights. Reference herein to any specific commercial product, process, or service by trade name, trademark, manufacturer, or otherwise does not necessarily constitute or imply its endorsement, recommendation, or favoring by the United States Government or any agency thereof. The views and opinions of authors expressed herein do not necessarily state or reflect those of the United States Government or any agency thereof. 


\section{Introduction}

In this paper, we shall report on some techniques for the development of preconditioners for the discrete systems which arise in the approximation of solutions to elliptic boundary value problems. These techniques are analysed in [11]. Here we shall only state the resulting theorems; complete proofs can be found in [11].

It has been demonstrated that preconditioned iteration techniques often lead to the most computationally effective algorithms for the solution of the large algebraic systems corresponding to boundary value problems in two and three dimensional Euclidean space (cf. [3] and the included references). The use of preconditioned iteration will become: even more important on computers with parallel architecture. This paper discussc's an approach for developing completely parallel multilevel preconditioners. In oreler to illustrate the resulting algorithms, we shall describe the simplest application of the technique to a model elliptic problem. Let $\Omega$ be a polygonal domain in $R^{2}$ and considen the problem of approximating the solution $u$ of

$$
\begin{aligned}
L u & =f \text { in } \Omega, \\
u & =0 \text { on } \partial \Omega,
\end{aligned}
$$

where

$$
L u=-\sum_{i, j=1}^{2} \frac{\partial}{\partial x_{i}} a_{i j} \frac{\partial u}{\partial x_{j}}+a u .
$$

We assume that the matrix $\left\{a_{i j}(x)\right\}$ is symmetric and uniformly positive definite: and $a(x) \geq 0$ in 5 .

We first define a sequence of multileve? finite element spaces in the usual way. Since $\Omega$ is polygonal, we can define a 'coarse' triangulation $\tau_{1}=U_{l} \tau_{1}^{l}$ where $\tau_{1}^{l}$ represents an individual triangle and $\tau_{1}$ denotes the triangulation. Successively finer triangulations $\left\{\tau_{k}, k=2, \ldots, J\right\}$ are defined by breaking each triangle of a coarser triangulation into four triargles by connecting the midpoints of the edges. The space $\mathcal{M}_{k}$ is defined to $x$

This manuscript, has been authored under contract number DE-AC02-76CH00016 with the U.S. Dopartment of Energy. Accordingly, the U.S. Government retains a non-exchusive, royalty-free licertisc lo publish or reproduce the published form of this contribution, or allow others to do so, for li.S. (ioverll. ment purposes. This work was also supported in part under the National Science Foundation (irant No. DMS84-05352 and by the U.S. Army Research Office through the Mathematical Science Lustinul.. Cornell University. 
the continuous functions defined on $\Omega$ which are piecewise linear with respect to $\tau_{k}$ and vanish on $\partial \Omega$. We shall be interested in developing a preconditioner for the solution of the Galerkin equations on the $J$ 'th subspace, i.e. $U \in \mathcal{M}_{J}$ satisfying

$$
A(U, \phi)=(f, \phi), \quad \text { for all } \phi \in \mathcal{M}_{J} \text {. }
$$

Here $A(\cdot, \cdot)$ denotes the generalized Dirichlet integral defined by

$$
A(u, v)=\sum_{i, j=1}^{2} \int_{\Omega} a_{i j} \frac{\partial u}{\partial x_{i}} \frac{\partial v}{\partial x_{j}} d x+\int_{\Omega} a u v d x
$$

and $(\cdot, \cdot)$ denotes the $L^{2}$ inner product on $\Omega$.

Let $\left\{\phi_{k}^{l}\right\}$ denote the usual nodal basis for the subspace $\mathcal{M}_{k}$, i.e. the l'th basis function is one on the l'th node of $k^{\prime}$ th triangulation and vanishes on all others. The preconditioner $\mathcal{B}$ is defined by

$$
\mathcal{B} v=\sum_{k=1}^{J} \sum_{l}\left(v ; \phi_{k}^{l}\right) \phi_{k}^{l} .
$$

The above preconditioner is simply a double sum, the terms of which can be computur! concurrently. This results in an inherently parallel algorithm.

As is well known, the rate of convergence of an iterative method can be estimated in terms of the condition number of the preconditioned system. In [11], a theory for the estimation of the condition number for this type of multilevel preconditioner in tornis of a number of a priori assumptions is given. In the above example, this theory can $l_{x}$ used to show that the relevant condition number is at worst $O\left(J^{2}\right)$. Moreover, these: results hold for problems in two, three and higher dimensions as well as problems with only locally quasi-uniform mesh approximation.

We note that many alternative preconditioning techniques have been propersed fen such discrete systems. For example, domain decomposition preconditioners have hern developed $([5],[6],[7],[8],\{14]$, and the included references). These domain denenufe)sition preconditioners are inherently parallel however become somewhat complex in three dimensional applications. Alternatively, multigrid $[4],[9],[15],[18]$ and hicharehi(al multigrid $[2],[21]$ techniques give rise to different multilevel preconditioner's. The standard multigrid algorithms do not allow for completely parallel computations since the computations on a given level use results from the previous levels. Theoretical results for the usual multigrid algorithms are available, in general, for problems in in! number of spatial dimensions but only for quasi-1uniform mesh approximation. (ion) results hold for the hierarchical basis method in two dimensions with refined mesilus l mul degenerate when applied to three dimensional problems. Finally, preconditioners bised on approximate LU factorization are often proposed however a comprehensive thery is yet to be developed [12], (13], [19].

The outline of the remainder of the paper is as follows. A general abstract therey for the development of parallel multilevel preconditioners is discussed in Section 2. In Section 3 , this theory is applied to second order elliptic boundary value problems and the serial and parallel complexity of the resulting algorithms is discussed. Wo inply 
the abstract theory to a second order problem with a locally refined mesh in Section 4. Finally, the results of numerical experiments illustrating the theory of the earlice sections are given in Section 5.

\section{General theory.}

In this section, we discuss a general theory for the construction of parallel multilevel preconditioners. This theory is presented in an abstract setting to most clearly illustrate the relevant analytic assumptions. The development of this class of preconditioner's is based on a certain orthogonal decomposition of the approximation space. The parallal multilevel preconditioners are then abstractly defined in terms of this decomposition l,y the replacement of orthogonal projections by more computationally efficient operatirs. Applications to second order elliptic boundary value problems are given in Sections 3 and 4 .

We start with the basic abstract framework. We assume that we are given a nested sequence of finite dimensional spaces,

$$
\mathcal{M}_{1} \subset \mathcal{M}_{2} \subset \ldots \subset \mathcal{M}_{J} \equiv \mathcal{M}, \quad J \geq 2
$$

The space $\mathcal{M}$ and hence all of its subspaces are equipped with two inner products $(\cdot, \cdot)$ and $A(\cdot, \cdot)$. The first part of this section will consider properties of a certain orthogonal decomposition of $\mathcal{M}$ with respect to the inner product $(\cdot, \cdot)$ and the secunence of sparcis (2.1). We shall investigate the spectral properties of these spaces with respect to the form $A(\cdot, \cdot)$ since, nltimately, we are interested in computing the solution to the Galcrkin equations: Given $f \in \mathcal{M}$, find $U \in \mathcal{M}$ satisfying

$$
A(U, v)=(f, v) \quad \text { for all } v \in \mathcal{M} \text {. }
$$

We shall use the following notation in the development. For each $k=1, \ldots, J$, w introluce the following operators:

(1) The projection $P_{k}: \mathcal{M} \longrightarrow \mathcal{M}_{k}$ is defined for $u \in \mathcal{M}$ by

$$
A\left(P_{k} u, v\right)=A(u, v), \quad \text { for all } v \in \mathcal{M}_{k} .
$$

(2) The projection $Q_{k}: \mathcal{M} \longrightarrow \mathcal{M}_{k}$ is defined for $u \in \mathcal{M}$ by

$$
\left(Q_{k} u, v\right)=(u, v), \quad \text { for all } v \in \mathcal{M}_{k}
$$

(3) The operator $A_{k}: \mathcal{M}_{k} \rightarrow \mathcal{M}_{k}$ is defined for $u \in \mathcal{M}_{k}$ by

$$
\left(A_{k} u, v\right)=A(u, v), \quad \text { for all } v \in \mathcal{M}_{k} .
$$

We shall also denote $A=A_{J}$ and define

$$
\mathcal{O}_{k}=\left\{\phi \mid \phi=\left(Q_{k}-Q_{k-1}\right) \psi, \psi \in M\right\}
$$


where $Q_{0}=0$. We shall discuss the spectral properties of $A$ with respect to the decomposition

$$
\mathcal{M}=\mathcal{O}_{1}+\cdots+\mathcal{O}_{J}
$$

It follows from the above definitions that

$$
\begin{aligned}
Q_{k} A & =A_{k} P_{k} \\
Q_{k} Q_{l} & =Q_{l} Q_{k}=Q_{l} \text { for } l \leq k .
\end{aligned}
$$

From the second equation of $(2.4)$, it follows that

$$
\left(Q_{k}-Q_{k-1}\right)\left(Q_{l}-Q_{l-1}\right)=0
$$

if $k \neq l$ and hence the decomposition $(2.3)$ is orthogonal, i.e. $(u, v)=0$ whenever $u \in \mathcal{O}_{l}, v \in \mathcal{O}_{k}$ with $l \neq k$.

We consider first the operator

$$
B=\sum_{k=1}^{J} \lambda_{k}^{-1}\left(Q_{k}-Q_{k-1}\right)
$$

where $\lambda_{k}$ denotes the spectral radius of $A_{k}$. Clearly, $B$ is symmetric and positive definitu and

$$
A(B A v, v)=\sum_{k=1}^{J} \lambda_{k}^{-1}\left\|\left(Q_{k}-Q_{k-1}\right) A v\right\|^{2},
$$

where $\|\cdot\|^{2}=(\cdot, \cdot)$. Note that $B$ is block diagonal with respect to the decomposition (2.3) and each diagonal block is a multiple of the identity matrix.

The operator $B$ may be thought of as an "approximate inverse" for $A$. Thus, wo shall state theorems estimating the condition number $K(B A)$ of $B A$. We note thinl $K(B A) \leq c_{1} / c_{0}$ for any positive constants $c_{0}, c_{1}$ satisfying

$$
c_{0} A(v, v) \leq A(B A v, v) \leq c_{1} A(v, v), \quad \text { for all } v \in M
$$

REMARK 2.1: The form of the operator $B$ can be motivated by the spectral decomposition of the operator $A$. Indeed, for a special example, namely, $\mathcal{M}_{k}$ the space spanned ly. the eigenvectors corresponding to the smallest $k$ distinct eigenvalues of $A$, the operator $B$ defined by $(2.5)$ is in fact equal to $A^{-1}$.

It is straightforward to show that ( (of. [11 1 )

$$
A(B A v, v) \leq J A(v v), \quad \text { for all } v \in \mathcal{M}
$$

The lower estimate of (2.7) will require some additional hypotheses concerning the spaces $\mathcal{M}_{k}$. We first consider the following assumptions on the operators $Q_{k}:$ For $k=1, \ldots, J$, there exists a constant $C_{1}>0$ such that,

$$
\left\|\left(I-Q_{k-1}\right) v\right\|^{2} \leq C_{1} \lambda_{k}^{-1} A(u, v), \quad \text { for all } v \in M .
$$

Wo have the following theorem and corollaries. 
Theorem 1. Assume that (A.1) holds. Then

$$
C_{1}^{-1} J^{-1} A(v, v) \leq A(B A v, v) \leq J A(v, v), \quad \text { for all } v \in \mathcal{M}
$$

Corollary 1. For any real s,

$$
B^{s}=\sum_{k=1}^{J} \lambda_{k}^{-s}\left(Q_{k}-Q_{k-1}\right) .
$$

Moreover, for any $s \in[0,1]$,

$$
J^{-s}\left(A^{s} v, v\right) \leq\left(B^{-s} v, v\right) \leq\left(C_{1} J\right)^{s}\left(A^{s} v, v\right), \quad \text { for all } v \in \mathcal{M}
$$

We have included Corollary 1 for the purpose of future applications which will not be described in this paper. In particular, it will be used for the development of preconclitioners for certain boundary operators which arise in domain decomposition technicues for second order boundary value problems [10].

In the next corollary, we consider the case of the sum of two operators. Let $A(\cdot, \cdot) \mid x$ another symmetric positive definite form and let $\hat{A},\left\{\hat{A}_{k}\right\}$ and $\left\{\hat{\lambda}_{k}\right\}$ be defined analogously in terms of $\hat{A}(\cdot, \cdot)$. Consider the operator $\vec{B}: \mathcal{M} \mapsto \mathcal{M}$ defined by

$$
\ddot{B}=\sum_{k=1}^{J}\left(\lambda_{k}+\hat{\lambda}_{k}\right)^{-1}\left(Q_{k}-Q_{k-1}\right)
$$

Theorem 1 immediately implies the following corollary.

Corollary 2. Assume that (A.1) holds for both A and A. Them,

$$
J^{-1}((A+\hat{A}) v, v) \leq\left(\ddot{B}^{-1} u, v\right) \leq C_{1} J((A+\hat{A}) v, v), \quad \text { for all } v \in \cdot M \text {. }
$$

The most natural application of the above corollary is to the liscrete systoms whinh arise in parabolic time stepping algorithms. At each time level, a function $l^{\prime \prime} \in . M$ satisfying

$$
(I+T \cdot A) U^{n}=F^{n}
$$

with known $F^{n} \in \mathcal{M}$ must be computed. Here $\tau$ is a positive number which is related to the time step size. We shall not consider further application of Corollary 2 in this paper.

We next apply the above results to analyze parallel multilevel preconditioners for $A$ An opexator $\mathcal{B}: \mathcal{M} \mapsto \mathcal{M}$ is a good preconditioner for $A$ if it satisfies:

(1) The action of $\mathcal{B}$ on vectors of $\mathcal{M}$ is economical to compute.

(2) The condition number $K(B A)$ of the preconditioned system is not too linger. 
Item (1) above guarantees that the cost per iteration in a preconditioned scheme using $\mathcal{B}$ for solving (2.2) will not be unreasonable. Item (2) guarantees that the number of itcrations in a preconditioned scheme will not be too large. Note that by Theorem $1, B$ satisfies (2). $B$ may in fact satisfy (1) in many applications but generally it is desirable to avoid evaluating the action of $Q_{k}$. Hence we shall develop more computationally effective algorithms by modifying (2.5).

To get a computationally effective preconditioner, we write $(2.5)$ in the form

$$
B=\sum_{k=1}^{J-1}\left(\lambda_{k}^{-1}-\lambda_{k+1}^{-1}\right) Q_{k}+\lambda_{J}^{-1} I
$$

Notice that if $\left\{\lambda_{k}\right\}_{k=1}^{J}$ satisfies the growth condition $\lambda_{k+1} \geq \sigma \lambda_{k}$, for $\sigma>1$ then the operator

$$
\hat{B}=\sum_{k=1}^{J} \lambda_{k}^{-1} Q_{k}
$$

satisfies

$$
\left(1-\sigma^{-1}\right)(\hat{B} u, u) \leq(B u, u) \leq(\hat{B} u, u), \quad \text { for all } u \in \mathcal{M}
$$

We consider a slightly more general operator defined by replacing $\lambda_{k}^{-1} I$ in $(2.12)$ with a symmetric positive definite operator $R_{k}: \mathcal{M}_{k} \mapsto \mathcal{M}_{k}$, i.e.

$$
\mathcal{B}=\sum_{k=1}^{J} R_{k} Q_{k}
$$

Clearly, $\mathcal{B}$ is symmetric and positive definite on $\mathcal{M}$. The cost of evaluating the action of the preconditioner $\mathcal{B}$ on a vector in $\mathcal{M}$ will be discussed in later sections bit will obvionsly depend on an appropriate choice of $R_{k}$.

For onr subsecquent development, we shall need to make the following assmundiun concerning the operator $R_{k}$. We assume that

$$
C_{2} \frac{\|u\|^{2}}{\lambda_{k}} \leq\left(R_{k} u, u\right) \leq C_{3}\left(A_{k}^{-1} u, u\right), \quad \text { for all } u \in \mathcal{M}_{k}
$$

where $C_{2}$ and $C_{3}$ are positive constants not depending on $J$. Clearly the choice $\Gamma_{k}=$ $\lambda_{k}^{-1} I$ corresponding to (2.12) satisfies (A.2).

The preconditioner (2.13) can be thought of as a parallel version of a V-cyele multimil algorithm. The operator $R_{k}$ plays the role of a smoothing procedure. The majou difference between (2.13) and the V-cycle multigrid scheme is that the smoothing (n) every level of (2.13) is applied to the original fine grid residual. In contrust, the multigrid V-cycle applies the smoothing to the residual computed using the corrections from the previously visited grid. Obvionsly, the different terms in (2.13) can be computed in parallel while, in contast, computations on a given grid level in a standarel multigriel algorithm must wait for the results from previous levels. The connection between (2.13) and the multigrid V-cycle will be more fully discussed in Sertion 3. Howerer, it is 
not surprising that assumptions which are equivalent to (A.2) have been made in the analysis of the ustal serial multigrid algoritims $[4],[9],[16],[17]$.

REMARK 2.2: A particularly interesting choice of $R_{k}$ can be motivated as follows. As noted above, $R_{k}=\lambda_{k}^{-1} I$ satisfies (A.2). Let $\left\{\psi_{k}^{\ell}\right\}$ be an orthonormal basis for $M_{k}$. Then

$$
\lambda_{k}^{-1} u=\lambda_{k}^{-1} \sum_{l}\left(u, \psi_{k}^{l}\right) \psi_{k}^{l}, \quad \text { for all } u \in \mathcal{M}_{k}
$$

In practice, an orthonormal basis for $\mathcal{M}_{k}$ is seldom available. However, for finite elenunt applications with quasi-uniform grids, the right hand side of $(2.14)$ with nommalianl nodal basis functions $\left\{\begin{array}{l}1 \\ k\end{array}\right\}$ defines an $R_{k}$ satisfying (A.2) (see Section 3). Moreorer. We note that for $u \in \mathcal{M}$,

$$
R_{k} Q_{k} u=\lambda_{k}^{-1} \sum_{i}\left(u, \bar{\psi}_{k}^{l}\right) \bar{\psi}_{k}^{l}
$$

and hence $R_{k} Q_{k}$ is computable without the solution of gram matrix systems. This will be discussed in more detail in Section 3.

With $\mathcal{B}$ defined in (2.13), we have the following corollary.

Corollary 3. Under assumptions (A.1) and (A.2),

$$
C_{1}^{-1} C_{2} J^{-1} A(v, v) \leq A(B . A v, v) \leq C_{3} J A(v, v), \quad \text { for all } u \in \mathcal{M}
$$

We next provide an altornative hypothesis for a lower estimate in (2.15). This is the so called "regularity and approximation" assumption often used in multigrid amalysin (cf. $[4],[15],[18]$ ). We assume that for a fixed $\alpha \in(0,1]$, there exists a positive constianl C. not depending on $k=1, \ldots, J$ satisfying

$$
A\left(\left(I-P_{k-1}\right) v, v\right) \leq\left(C_{A} \lambda_{k}^{-1}\left\|A_{k} v\right\|^{2}\right)^{\alpha} A(v, v)^{1-\alpha}, \quad \text { for all } u \in \mathcal{M}_{k}
$$

where $P_{0}=0$. In finite element applications, the above assumption is usually proverl $b$ : using elliptic regularity for the continuous problem and the approximation properticis of the space $\mathcal{M}_{k-1}[1],[4]$. In such applications, assumption (A.3) naty be stronger thim (A.1), (.g. when $\alpha=1,(A .3)$ implies (A.1).

Theorem 2. Assume that (A.2) and (A.3) hold. Then

$$
C_{2} C_{1}^{-1} J^{1-1 / n} A(u, v) \leq A(B A v, u) \leq C_{3} J A(v, v), \quad \text { for all } u=\mathcal{M}
$$

Remark 2.3: Included in $(A .1)$ and $(A .3)$ is the implicit assumption that $C_{1}$ and $C_{1}$ are greater than or equal to $K\left(A_{1}\right)$. In finite element applications, $K\left(A_{1}\right)$ will uot h large if the grid size of the coarsest grid is of unit size. However, if a good preconditionut $R_{j}$ is avalable for any finer grid, i.e. $R_{j}$ satisfies in addition,

$$
\left(R_{j}^{-1} u, u\right) \leq C_{5}\left(A_{j} u, u\right)
$$

then it suffices to use

$$
\mathcal{B}=\sum_{k=j}^{J} R_{k} Q_{k}
$$

In such applications, (A.1) or (A.3) need only be satisfied for $k>j$. Note that $R_{j}=., 1$ will be computationally economical provided that the $j$ th grid size is relativaly small. Many alternative choices are possible. 


\section{The quasi-uniform finiet element application.}

In this section, we shall illustrate the application of the abstract theory and algorithin:; discussed in the previous section to a second order elliptic boundary value problem approximated using finitr element functions on a quasi-uniform mesh. We note that. the hypotheses of the previous section are satisfied. We also consider the computational complexity of the resulting algorithm in both serial and parallel computing applications. For brevity, we consider only the most basic finite element applications. Many otlick applications are possible including examples of elliptic problems in higher dimensions.

Let $\mathcal{M}_{1} \subset \cdots \subset \mathcal{M}_{J} \equiv \mathcal{M}$ be the finite element spaces defined in the introduction subsequent to $(1.1), A(\cdot, \cdot)$ be the generalized Dirichlet form defined in (1.3) and $(\cdot, \cdot)$ $b$, the $L^{2}$ inner product on $\Omega$.

We will apply the results stated in Section 2 to Problem (1.2) with the above seculuncu of spaces. Let $h_{k}$ denote the size of the $k^{\prime}$ th triangulation. It easily follows that threr are constants $c_{0}$ and $c_{1}$, not depending on $k$ and satisfying

$$
c_{0} h_{k}^{-2} \leq \lambda_{k} \leq c_{1} h_{k}^{-2}
$$

Inequality (A.1) with $k>2$ is well known. For $k=1$, we have that

$$
\|v\|^{2} \leq \Lambda^{-1} A(v, v), \quad \text { for all } v \in \mathcal{M}
$$

where $A$ is the smallest eigenvalue of $A$ and is obviously bounded from away from acen (independently of $J$ ). We shall suppose in this application that $\mathcal{M}_{1}$ is such that $h_{1}$ in proportional to the diameter of $\Omega$ so that $C_{1} \geq \lambda_{1} / \Lambda$ which is not large.

We next consider the operator $R_{k}$ motivated by Remark 2.2 , i.e.

$$
R_{k} v=\sum_{1}\left(v, \phi_{k}^{l}\right) \phi_{k}^{l}, \text { for } v \in \mathcal{M}_{k}
$$

where the sum is taken overe all nodes of $\tau_{k}$. As observed in Remank 2.2, the netion ol $R_{k}\left(Q_{k}\right.$ can be computed without explicitly computing $Q_{k}$. Morenver, using $R_{k}$ retines by $(3.2)$ in (2.13) leads to the preconditioner of $(1.4)$ It is shown in $[11]$ that $(A .2)$ ' for this $R_{k}$.

For this problem, (A.3) will always be satisfied for some $\alpha \in(0,1]$, (cf. $[1],[4]$ ). T'lu size of $\alpha$ depends on the elliptic regularity of Problem (1.1). Thus, in the case when!? is a convex polygonal domain and the coefficients defining $L$ are smooth, (A.3) holdis with $x=1$ and we conchude from Theorem 2 that

$$
\Pi(\mathcal{B}, A) \leq c . J
$$

In the case of a so called crack problem (with smooth coefficients), the largest interiof angle is $2 \pi$ and the regularity of $(1.1)$ is such that. (A.3) does not hold for $a \geq 1 / 2$. Hence Corollary 3 yields the better estimate and shows that

$$
I(\mathcal{B} . A) \leq C \cdot J^{2}
$$


Remark 3.1: It is possible to apply the theory of Section 2 to elliptic problems in thre or more dimensions. Many examples are possible but we consider only the simplest. In three dimensions, we let the coarse mesh be a union of equally sized cubes. Finer mushus are obtained by breaking each cube of a conser mesh into eight smaller cubes in the: obvious way. The subspaces $\left\{\mathcal{M}_{k}\right\}$ are defined to be the functions on $\Omega$ which are continuous and piecewise trilinear with respect to the $k^{\prime}$ th mesh and vanish on $\partial \Omega$. The nodes of these spaces are the vertices of the cubes defining the mesh. We may take

$$
\mathcal{B} u=\sum_{k=1}^{J} h_{k}^{-1} \sum_{l}\left(u, \phi_{k}^{l}\right) \phi_{k}^{l},
$$

where $\left\{\phi_{k}^{\prime}\right\}$ denotes the set of nodal basis functions. We emphasize here again that all the terms in (3.3) are independent and hence may be computed concurrently.

Remark 3.2: Assumption (A.1) is often easier to verify than (A.3). For exampic, w' consider the two dimensional problem (1.1) when the coefficients of the operator $L$ ur. discontinuous. If the jumps in the coefficients are only along the lines of the coursc mesh, then it is possible to prove that (A.1) holds with $C_{1} \leq C^{\prime} J$ where the constant $C$ depends on the local variation of the coefficients of $L$ on the coarse grid triangles linl not on the magnitude of the jumps across triangles [20]. This leads to a conditionium restilt of the form

$$
K(\mathcal{B} .4) \leq C^{\prime} \cdot J^{3}
$$

The dependence of constant $C_{1}($ in $(A, 3))$ on the size of the jumps is a much more di ti cult question since it requires the knowledge of the dependence of the elliptic regulanity constants on such jumps.

In the remainder of this section, we consider computational issues involved in inple. menting the a! ove algorithm in serial and parallel computing architectures. Howerer. before proceeding, we make the following observation. Even thongh we have detind

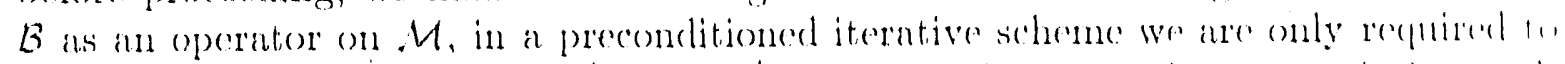

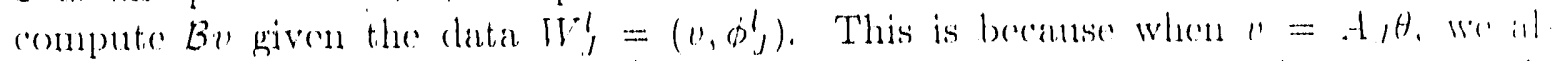

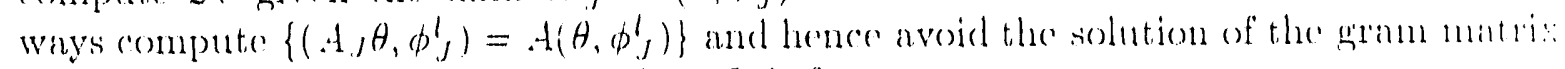
problem recpuired for the computation of $A, \theta$.

We first consicler the serial version of the algorithen. Let $\theta \in . M$ be given anm define $W_{k}^{l}=\left(v, \phi_{k}^{l}\right)$. Let $W_{k}$ denote the vector with entries $\left(W_{k}\right)_{l}=W_{k}^{l}$. We nen to compute the action of $\mathcal{B} v$ given $W_{J}$. Wo define $W_{k-1}$ from $W_{k}^{r}$ in a recursive manner. Note that cach basis function in $M_{k-1}$ can be written as a local lineng combination of busis functions for $\mathcal{M}_{k}$. Thus, anch value of $W_{k-1}^{l}$ can be written ats a local lineite combination of values of $W_{k}$. Moreover, the work involved in computing $F_{k-1}$ froul $\|_{k}$ is proportional to the number of unknowns in $M_{k-1}$. Consequently, the work involind in computing the vectors $\left\{W_{k}\right\}, k=1, \ldots, J$ bounded by a constant tinnes the munnlen of unknowns in $\mathcal{M}$. Once the vectors $\left\{W_{k}\right\}$ are known, we are left to compute the representation of $\mathcal{B} v$ in the besis for. M. 'To do this, we compute the representiation al'

$$
\mathcal{B}_{m} v \equiv \sum_{k=1}^{m} \sum_{1}\left(v, \phi_{k}^{l}\right) \phi_{k}^{l},
$$


in the basis for $\mathcal{M}_{m}$, for $m=1, \ldots, J$. The result at $m=J$ is of course the basis representation for $\mathcal{B} v$. For $m=1$, the representation is already given by $W_{1}$. The representation of $\mathcal{B}_{m} v$, for $m>1$ is calculated from that of $\mathcal{B}_{m-1} v$ by interpolating the $\mathcal{B}_{m-1} v$ results (i.e. expanding them in terms of the $m$ 'th basis ) and adding the m'til level contribution from $W_{m}$. The work of calculating the representation of $\mathcal{B}_{m} v$ giv'n that for $\mathcal{B}_{m-1} v$ is on the order of the number of unknowns in $\mathcal{A}_{m}$ and thus the totul work for this algorithm is bounded by a constant times the number of unknowns on the finest grid.

Remark 3.3: The scrial implementation of the operator $\mathcal{B}$ is closely related to 1 la. multigrid V-cycle algorithm. The step of computing $W_{k-1}$ from $W_{k}$ is nothing mon. that the step which "transfers the residuals" from grid level $k$ to $k-1$ in a multigricl $\mathrm{V}$-cycle algorithm. However, the multigrid algorithm requires extra computation since it must smooth and then compute new residuals on the $k$ 'th level before transferring. The second step in the serial algorithm for $\mathcal{B}$ is also duplicated in the "coarser to finc" interpolation" step in the multigrid V-cych: algorithm. The symmetric multigrid ${ }^{\prime}$ cycle requires extra computation since it requires additional smoothing on each grid level. Thus the setial $\mathcal{B}$ algorithm, in terms of complexity, is similar to a multigrivl $\mathrm{V}$-cycle algorithm without smoothing.

We next consider parallel implementation of the preconditioner $\mathcal{B}$. The execution of $(1.4)$ can obviously be made parallel in many ways by broaking up the terms into various numbers of parallel tasks. The optimal splitting of the sum is clearly dependen! on characteristics of the individual parallel computer, for exmple, nemory manamenum considerations, task initialization overhead, the number of parallel processors, (1.c. IV. note, however, the simplicity of the form of (1.4) allows for almost complete frecelun for parnllel splitting.

It is of theoretical interest to consider the algorithm on a shared memory manchin. with an unlimited number of processors. As above, the implementation $\mathcal{B} v$ involves twe steps, the calculation of the coefficients $W_{k}^{l}$ and the compratation of the represcontation of $B$, in the basis for $M$. Ench coefficient can be computed independently and involve... a linear combination (not necessarily local) of the values of $W_{J}$. With enomgh pures. sors, a lincar combination of $m$ numbers can be computed in $\log _{2}(m)$ time. Hence the confficient vectors $\left\{W_{k}\right\}$ can be computed in $\log _{2}(N)$ time where $N$ is the dimension of $M$. Each coefficient of $\mathcal{B} v$ involves a linear combination of $M_{n} J$ contributions from $\|_{1}$ .$J$ grid levels (here $M_{n}$ is the maximum number of neighbors for any given level. Thut. computation of $\mathcal{B} u$ can in clone in time bounded by $C . J$.

\section{A local refinement application.}

In this section, we shall discuss the application of the parallel multilevel algorithun wo the finite element equations corresponding to a problem with mesh refinement. Sucls mesh refinements are necessary for accurate nodeling of problems with varions trye of singrular behavior. For simplicity, wo shall make no attempt at generality. Instracl. we shall illustrate the technigue by considering an example from which many obrimu: genoralizations are pessible. For this example, the domain $\Omega$ will be the unit stuma and wo shall approximate the solution to $(1.1)$. The form $A(\cdot, \cdot)$ and the inner produre 
$(\cdot, 1)$ will be as in Section 3. The sequence of grids which we shall consider will h progressively more refined as we approach the corner $(1,1)$. Such a mesh would ha effective if, for example, the function $f$ in (1.1) behaved like a $\delta$ function distrilmuinu at the point $(1,1)$.

To define the mesh, we first start with a sequence of subspaces $\mathcal{M}_{1}, \ldots, \mathcal{M}_{j}$ defined using uniform grids of size $h_{k}=2^{-k}, k=1, \ldots, j$ as described in the quasi-uniform case (See Section 1). The $j+1$ 'st triangulation is then defined by refining only those triangles in the upper quarter, $[1 / 2,1] \times[1 / 2,1]$. Similarly, the $j+2$ 'nd triangulation is clefined by rofining only those triangles in the $j+1$ 'st grid which are in the lowion $[3 / 4,1] \times[3 / 4,1]$, etc. Accordingly, wo shall denote $\Omega_{k}=\left(1-2^{j-k}, 1\right) \times\left(1-2^{j-k}\right.$. 1$)$ inlul define the spaces $\mathcal{M}_{k}$ for $k=j+1, \ldots, J$ to be the continuous functions on $\Omega$ whinh ar. piecewise linear with respect to the $k^{\prime}$ th grid. Note that this introduces slave nodes int., the computation, i.e. the vertices of the triangles on the boundary of the k'th refincmen region which are not nodes for the $k-1$ 'st subspace. These nodes are slaves since the: values of functions on these nodes are determined by the values of neighboring noctus and the continuity condition on the subspace. Thus, they do not represent degrees of freedom in the subspace. It is shown in [11], that (A.1) is satisfied for this secunctur' of subspaces.

We next define a secquence of operntors $\left\{R_{k}\right\}$ satisfying (A.2). For $k \leq j, \Gamma_{k} i_{i}$ given by (3.2). Let $\left\{x_{k}^{i}\right\}$ denote the nodes of the $k^{\prime}$ th grid and let $\left\{\phi_{k}^{l}\right\}$ denote Ho. corresponding nodal basis functions. For ench node $i k$ with $k>j$ we define

$$
h_{k l}= \begin{cases}h_{k} & \text { if } x_{k}^{l} \in \bar{\Omega}_{k}, \\ h_{m} & \text { if } x_{k}^{l} \in \bar{\Omega}_{m} / \bar{\Omega}_{m+1}, j \leq m<k .\end{cases}
$$

Note that if $x_{k}^{l} \in \bar{\Omega}_{k} / \bar{\Omega}_{k+1}$ then $x_{k}^{l}$ is a node for cach finer subspace and gets assignuel the same value h.k. We then clefine

$$
R_{k} u=h_{k}^{2} \sum_{l} h_{k l l}^{-2}\left(u, \phi_{k}^{l}\right) \phi_{k}^{l} .
$$

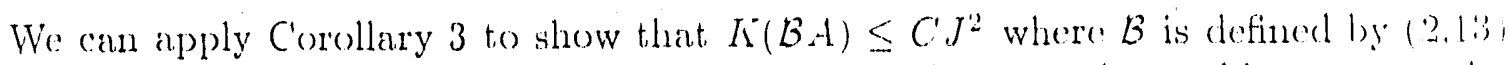
with $R_{k}$ and $\mathcal{M}_{k}$ as above. For this application, we have not becn able to prore l regularity and approximation assumption (A.3).

For the prupose of implementation, it is more efficient to reorder the terms definine B. For $k=j, \ldots$. J let $\mathcal{N}_{k}$ be the nodes of $\mathcal{M}_{k}$ in $\Omega_{k}$ and for $k<J$ let $\mathcal{N}_{k}^{1}$ be the nockes of $\mathcal{M}_{k}$ in $\widetilde{\Omega}_{k} / \Omega_{k+1}$. For a function $u \in \mathcal{M}$, it is not difficult to see by induction on .J that

$$
\begin{aligned}
\mathcal{B} u & =\sum_{k=1}^{j-1} R_{k} Q_{k} u+\sum_{x_{J}^{\prime} \in N_{l}}\left(u, \phi_{J}^{l}\right) \phi_{J}^{\prime} \\
& +\sum_{k=j}^{J-1}\left[\sum_{x_{k}^{\prime} \in \mathcal{N}_{k}^{1}} \gamma_{k}^{J}\left(u, \phi_{k}^{l}\right) \phi_{k}^{l}+\sum_{x_{k}^{\prime} \in \mathcal{N}_{k} / \mathcal{N}_{k}^{\prime}}\left(u, \phi_{k}^{l}\right) \phi_{k}^{l}\right]
\end{aligned}
$$


where $\gamma_{k}^{J}=h_{k}^{-2} \sum_{m=k}^{J} h_{m}^{2}$. Note that the $R_{k}$ terms in the first sum of (4.2) involves tho same sums which appear in the uniform case of Section 3. In addition, the calculation corresponding to the $k$ 'th mesh in $(4.2)$ for $k=j, \ldots, J$ only involves nodal basis functions on $\bar{\Omega}_{k}$.'

Finally we define a simpler preconditioner $\hat{\mathcal{B}}$ by replacing $\gamma_{k}^{J}$ by one in (4.2), i.e.

$$
\hat{\mathcal{B}} u=\sum_{k=1}^{j} \sum_{l}\left(u, \phi_{k}^{l}\right) \phi_{k}^{l}+\sum_{k=j+1}^{J} \sum_{x_{k}^{l} \in \mathcal{N}_{k}}\left(u, \phi_{k}^{l}\right) \phi_{k}^{l} .
$$

Note that in $(4.3)$ the $k$ 'th refinement grid only adds a sum over the nodes in $\bar{\Omega}_{k}$. Wr. note that for $u \in \mathcal{M}$, by (4.2)

$$
\begin{aligned}
(\mathcal{B} u, u)= & \sum_{k=1}^{j-1} \sum_{l}\left(u, \phi_{k}^{l}\right)^{2}+\sum_{x_{J}^{l} \in \mathcal{N}_{J}}\left(u, \phi_{J}^{l}\right)^{2} \\
& +\sum_{k=j}^{J-1}\left[\sum_{x_{k}^{l} \in \mathcal{N}_{k}^{1}} \gamma_{k}^{J}\left(u, \phi_{k}^{l}\right)^{2}+\sum_{x_{k}^{l} \in \mathcal{N}_{k} / \mathcal{N}_{k}^{1}}\left(u, \phi_{k}^{l}\right)^{2}\right]
\end{aligned}
$$

with an analogous expression for $\hat{\mathcal{B}}$. Clearly, $1 \leq \gamma_{k}^{J} \leq 4 / 3$ from which it follows that

$$
(\hat{\mathcal{B}} u, u) \leq(\mathcal{B} u, u) \leq \frac{4}{3}(\hat{\mathcal{B}} u, u), \quad \text { for all } u \in \mathcal{M} \text {. }
$$

From the discussion in Section 3, it is clear that the first sum in (4.3) is a preconclitioner for the problem on $\mathcal{M}_{j}$, i.e. the finest uniform grid. As we shall see, this sum call also be replaced by any uniform preconditioner for $A_{j}$ without adversely effecting the asymptotic behavior of the overall condition number. Indeed, let the operator $R_{j} l_{x}$ il preconditioner for $A_{j}$ (satisfying (2.16) and the second inequality of $(A .2)$ ) and ditiu. for $u \in \mathcal{M}$,

$$
\hat{\bar{B}} u=R_{j} Q_{j} u+\sum_{k=j+1}^{J} \sum_{x_{k}^{l} \in \mathcal{N}_{k}}\left(u, \phi_{k}^{l}\right) \phi_{k}^{l} .
$$

Note that by Remark 2.3, the operator

$$
\tilde{B} u=\sum_{k=j}^{J} R_{k} Q_{k} u
$$

satisfies $K(\tilde{B} A) \leq C(J-j)^{2}$. It is shown in [11] that $\tilde{B}$ is uniformly equivalent to $\dot{B}$. Thus, $K(\hat{B} . A) \leq C(J-j)^{2}$.

Remark 4.1: Clearly, we could generalize this example to include much more genclail refinements for problems in $R^{2}$ as well as higher dimensional space. Note that the refinement only changes the preconditioner $\hat{\mathcal{B}}$ (resp. $\hat{B}$ ) by adding additional terms in 
(4.3) (resp. (4.4)) involving nodes from the refinement region. Thus, this approilch is well suited to dynamic adaptive refinement techniques. New refinement regions adrl terms to the sum whereas the "de-refinement" of existing regions only takes away tolmis from the sum. The operator $\dot{B}$ is even more useful in this context since it allows the asy. inclusion of this refinement preconditioner into existing large scale uniform grid colcs. Preconditioners for the uniform grid already available in the existing code can be used. supplemented with additional routines implementing the terms due to the refinement.

\section{Numerical results}

In this section, we provide the results of numerical examples illustrating the then!" discussed in the earlier sections. To demonstrate the performance of the proposed al. gorithms, we shall provide numerical results for a two dimensional problem with ful! elliptic regularity and one with less than full elliptic regularity, a two dimensional ( $x$ ample with a geometric mesh refinement and a three dimensional example. In all of the reported results, the experimentally observed behavior of the condition number of the preconditioned system was in agreement with the theorems presented earlier. In the first example, we also compare the results of the new method with those olstainer using the hierarchical preconditioning method [21] and a classical V-cycle multimit preconditioner [4].

For our first example, we consider Problem (1.1) when $L=-\Delta \equiv-\partial^{2} / \partial x_{1}^{2}-\partial^{2} / \partial x^{2}$ and $\Omega$ is the unit square. This example satisfies the regularity and approximation assumption (A.3) for $\alpha=1$ as well as (A.1).

We will use a finite element discretization of (1.1) and develop a sequence of grilin in a standard way. To define the coarsest grid, we start by breaking the squitre into four smaller squares of side length $1 / 2$ and then dividing each smaller square into tiw triangles by connecting the lower left hand corner with the upper right hand cormel. Subsequently finer grids are developed as in the introduction, i.e., by dividing ("ill triangle into the fou triangles formed by the edges of the original triangle anil $1 \mathrm{~h}_{1}$ lines connecting the centers of these edges. The space $\mathcal{M}_{i}$ is detined to be the sin of continuous functions on $\Omega$ which are piecewise linear on the $i$ 'th triangulation ant vanish on $\partial \Omega$.

We shall compare three pieconditioners for (1.2). The first preconditioner $B$ is detined by the multilevel algorithm (2.13) with $R_{k}$ given by $(3.2)$ and fits into the frameworl: consivered in Section 3. For comparison, we also provide results for the hierarelical preconditioner $B_{H}[\mathbf{2 1}]$ and a preconditioner $B_{M}$ defined by a standard symmetric $l$. cycle of multigrid [4]. The multigrid algorithm uses one sweep of Jacobi smonthine whenever a grid level is visited and hence results in two smoothing steps on ciale grit for each evaluation of the preconditioner. The multigrid uses $h_{0}=1 / 4$ for the counstis grid while both the hierarchical and the parallel multilevel algorithms uses $h_{0}=1 / 2$.

Table 5.1 gives the condition numbers $K$ of the preconditioned systems $B_{H} A, B . A$ ind $B_{M} A$ corresponding, respectively, to the hierarchical preconditioner, the preconditione' defined by (2.13), and the V-cycle multigrid preconditioner. We note that for these examples, a preconditioned conjugate gradient algorithm 1 ing the new preconditione would be expected to take twice as many iterations as the corresponding algorithu using the V-cycle of multigrid. However, even in a serial implementation, the multiminl 
a.lgorithm involves substantially more computational effort per step. The new mothinl outperforms the hierarchical preconditioner.

Table 5.1

Condition numbers when $\Omega$ is the square.

\begin{tabular}{|c|c|c|c|}
\hline$h_{J}$ & $K\left(B_{H} A\right)$ & $K(\mathcal{B} A)$ & $K\left(B_{M} A\right)$ \\
\hline $1 / 16$ & 19 & 7.0 & 2.3 \\
$1 / 32$ & 31 & 8.1 & 2.4 \\
$1 / 64$ & 43 & 9.0 & 2.4 \\
$1 / 128$ & 58 & 9.8 & 2.4 \\
\hline
\end{tabular}

This test problem illustrates an example where all three methods work reasonably. well. However, we note that $\mathcal{B}$ is preferred over standard multigrid when the parallel aspects of the algorithm are important. In addition, $\mathcal{B}$ generalizes to higher dimensional problems without convergence rate deterioration (see Table 5.5) and hence would br: preferred to the hierarchical method in three dimensional computations.

We next consider the above preconditioners on a problem with less than full ollipiti. regularity. We again consider (1.1) with $L$ given by the Laplacian and $\Omega$ equal to thr "slit domain", i.e. $\Omega$ is the set of points in the interior of the unit square exchuling the line $\{(1 / 2, y) \mid y \in[1 / 2,1)\}$. This example cloes not satisfy the a priori estimatces niscl in the proof of the regularity and approximation assumption (A.3) for $\alpha \geq 1 / 2$. Howerer. assumption (A.1) is satisfied.

Table 5.2 gives the condition numbers $I$ of the preconditioned systems $B_{H} A, \mathcal{B} A$ and $B_{M} A$ corresponding, respectively, to the hierarchical preconditioner, the preconditionet defined by (2.13), and the V-cycle multigrid preconditioner. The results are in gencril agreement with the theoretical estimates

$$
\begin{aligned}
H^{2}\left(B_{H} A\right) & \leq C \ln ^{2}\left(1 / h_{\jmath}\right), \\
H(B A) & \leq C \ln ^{2}\left(1 / h_{,}\right),
\end{aligned}
$$

for the respective methork.

Table 5.2

Condition numbers when $\Omega$ is the slit domain.

\begin{tabular}{|c|c|c|c|}
\hline$h . J$ & $\Lambda^{\prime \prime}\left(B_{H} A\right)$ & $\Pi(\mathcal{B} A)$ & $\Pi(B, A)$ \\
\hline $1 / 16$ & 14.6 & 7.9 & 2.6 \\
$1 / 32$ & 25.17 & 10.0 & 2.9 \\
$1 / 64$ & 38.2 & 12.6 & 3.1 \\
$1 / 128$ & 53.8 & 14.9 & 3.4 \\
\hline
\end{tabular}

We next provide numerical results for the refinement example of Section 4 . Wo once again, consider the solution of (1.1) with $L$ the Laplacian and $\Omega$ the unit square. Thu sequence of spaces $\mathcal{M}_{:} \subset \cdots \subset \mathcal{M}_{J}$ are as developed in Section 4 and provide resultis 
for the preconditioner $\hat{\mathcal{B}}$ defined by (4.3). As noted in Section 4, some such refinc'mlun would be necessary if, for example, the function $f$ had a $\delta$-function behavior at the point $(1,1)$. Table 5.3 gives the condition number of the preconditioned system 2.4 is a function of the mesh size of the uniform grid $h_{j}$ and the number of refinement liver. l. The size of the finest triangle can be computed by dividing the uniform mesh size liy. $2^{l}$. In all of the "uns, the coarsest grid level corresponded to $h_{0}=1 / 2$. The numericil resulis seem to undicate that an increase in the number of uniform levels has a greitir. effect on the condition number than an increase in the number of refinement levels.

Table 5.3

Condition numbers for the refinement exumple.

\begin{tabular}{|c|c|c|c|c|}
\hline$h_{j}$ & $l=1$ & $l=2$ & $l=3$ & $l=4$ \\
\hline $1 / 8$ & 6.3 & 6.5 & 6.7 & 6.9 \\
$1 / 16$ & 7.7 & 7.9 & 8.05 & 8.1 \\
$1 / 32$ & 8.8 & 9.0 & 9.1 & 9.2 \\
$1 / 64$ & 9.6 & 9.7 & 9.8 & 9.9 \\
\hline
\end{tabular}

We next present results for the refinement operator defined by (4.4). The prolikiti and sequence of subspaces are as just described but only the subspaces $\mathcal{H}_{k}, k \geq i$ in' used. In (4.4), we use a multigrid preconditioner (cf. [4]) scaled by 4 to define $R$, the operator on the finest uniform grid. The scaling was introduced to bilance the siar of the two terms in (4.4). Table 5.4 gives the condition number of the preconditionul system $\hat{B} A$ as a function of the mesh size of the uniform grid $h_{j}$ ard the number of refinement levels $l$.

Table 5.4

Condition numbers for $\hat{B} A$ using multigrid precondationing on level $j$.

\begin{tabular}{|c|c|c|c|c|}
\hline$h_{j}$ & $l=1$ & $l=2$ & $l=3$ & $l=4$ \\
\hline $1 / 8$ & 4.3 & 6.0 & 6.4 & 6.6 \\
$1 / 16$ & 4.7 & 6.7 & 7.6 & 8.1 \\
$1 / 32$ & 4.9 & 7.0 & 8.4 & 9.2 \\
$1 / 64$ & 5.0 & 7.1 & 8.5 & 9.6 \\
\hline
\end{tabular}

As a final example, we illustrate the preconditioning technicue on a threc alinemsinum! problem. We consider a Galerkin approximation to the Laplace efuation

$$
\begin{aligned}
-\Delta u & =f \text { in } \Omega, \\
u & =0 \text { on } \partial \Omega,
\end{aligned}
$$

where $\Delta=\partial^{2} / \partial x^{2}+\partial^{2} / \partial y^{2}+\partial^{2} / \partial z^{2}$ and $\Omega$ is the unit cube. We lefine the coitro mesh by dividing $\Omega$ into eight smaller cubes of size $h_{0}=1 / 2$. Successively finct meshen are formed by dividing each cube of a coarser mesh into eight smaller cubes. The fiutu 
element space $\mathcal{M}_{k}$ is defined to be the set of continuous functions on $\Omega$ which ur. trilinear with respect to the $k^{\prime}$ th mesh and vanish on $\partial \Omega$.

Table 5.5 gives the condition number $I$ of the preconditioned system $\mathcal{B}$ A whore $L^{\circ}$ is defined by (3.3). This example satisfies full elliptic regularity and the regulurit! and approximation assumption (A.3) holds with $\alpha=1$. Thus, the theory predictis only a logarithmic growth in the condition number which is in agreement with the reported results. Note the finite element spaces are of rather large dimension, in fin't. the $h_{J}=1 / 64$ example has over a quarter of a million unknowns.

Table 5.5

Condition numbers for the three dimensional example.

\begin{tabular}{|c|c|}
\hline$h_{j}$ & $K(\mathcal{B} A)$ \\
\hline $1 / 8$ & 4.1 \\
$1 / 16$ & 5.2 \\
$1 / 32$ & 6.0 \\
$1 / 64$ & 6.6 \\
\hline
\end{tabular}

\section{References}

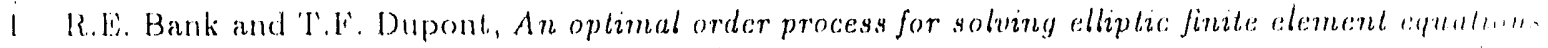
Wath, Comp. 36 (1981), 35- -51.

2. R.E. Bank, T.F. Dupont, and HI. Yserentant, The hierarchical basis multagrad method, Num1. Manll, $52(1988), 427-458$.

3) (a. Birkhoff and A. Schoenstad, eds, "Elliptic Problem Solvers II," Academic Press, Now Yinl. 1984.

t J.H. Bramble and J.E. Pasciak, New convergence estimates for multigrid rlgorithms, Math. ('illi). $49(1987), 311-329$.

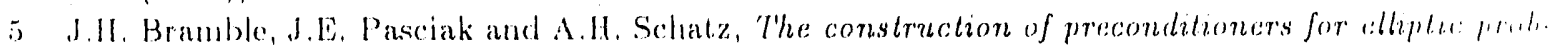
lems by substructuring, l, Malh. Comp. 47 (1986), 103.134.

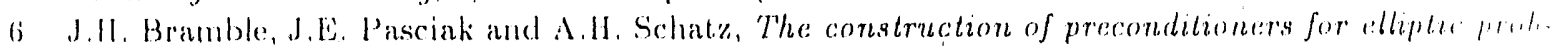
lems by substructuring, II, Math. Comp. 49 (1987), 1⒗

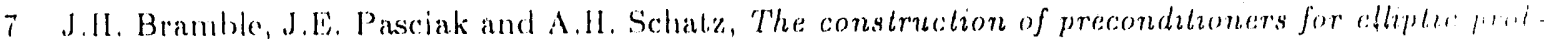
lems. by substructuring, III, Math. Comp, 51 (1988), $415 \cdots 430$.

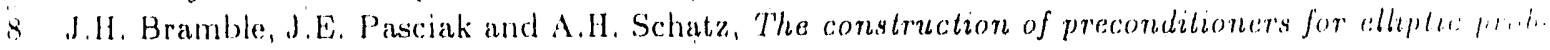
lems by substructuring, IV, Math. Comp. (to appear).

9) J.H. Bramble, J.E. Pasciak and J. Xu, The analysis of multigrid algorthms with non-nestet spm... or non-inherited quadratic forms, Math. Comp., (submitted).

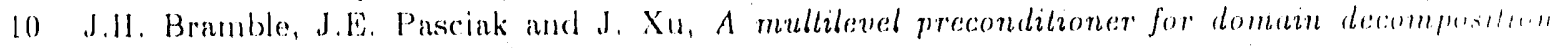
boundary systems, (in proparation).

11 J.H. Bramble, J.E. Pasciak and J. Xu, Parallel multilevel preconditioners, Mat.h. Connp., (sil) t.ed).

12 P. Concus, G.II. Golub and G. Meurant, Block preconditioning for the conjugale gradient melluwl. SIAM J. Sci. Stat. Comput. 6 (1985), 220-252.

is Dupont, T., Kendall, R.P., and Rachford, H.H.,. An approximale factorization proctsdm l...

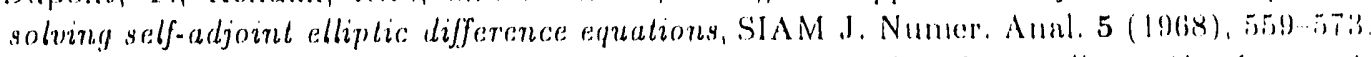

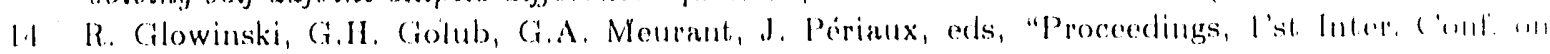
Domain Decomposition Methods," SIAM, Philadelphin, 1988.

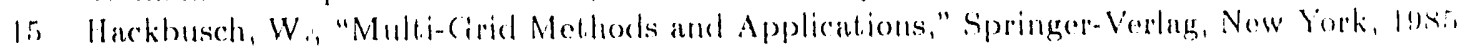


16 S. F. McCormick, Multigrid Methods for Variational Problems: Further Results, SIAN J. Vullu1 Anal, 21 (1984), $255-263$.

17 S. F'. McCormick, Multigrid Methods for Variational Problems: General Theory for the V-cinels. SIAM J. Numer. Anal, 22 (1985), 634-643.

18 J. Mandel, S. McCormick and R. Bank, Variational multigrid theory, in "Multigrid Molliols," Ed. S. McCormick, SIAM, Philadelphia, Penn., pp, 131-178.

19 J.A. Meyorink and H.A. van'der Vorst, Iterative methods for the solution of linear system. (1) which the coefficient matrix is a symmetric M-matrix, Math. Comp. 31 (1977), 148-162.

20 J. Xu, "Theory of Multilevel Methods," Cornell Univ. (Thesis), 1988.

21 H. Yserentant, On the multi-lavel splitling of finite element spaces, Numerische Malhoualik 19 $(198(6), 379-412$.

Author's Addresses

Cornell University

Ithaca, N.Y. 14853

E-mail: bramble@mssun7.msi.conell edu

Brookhaven National Laboratory

Upton, N.Y. 11973

E-mail: pasciak@bnl.gov

Pennsylvania State University

University Park, Penn. 16802

H-mail: xu(G) rayleigh.psu.edu 

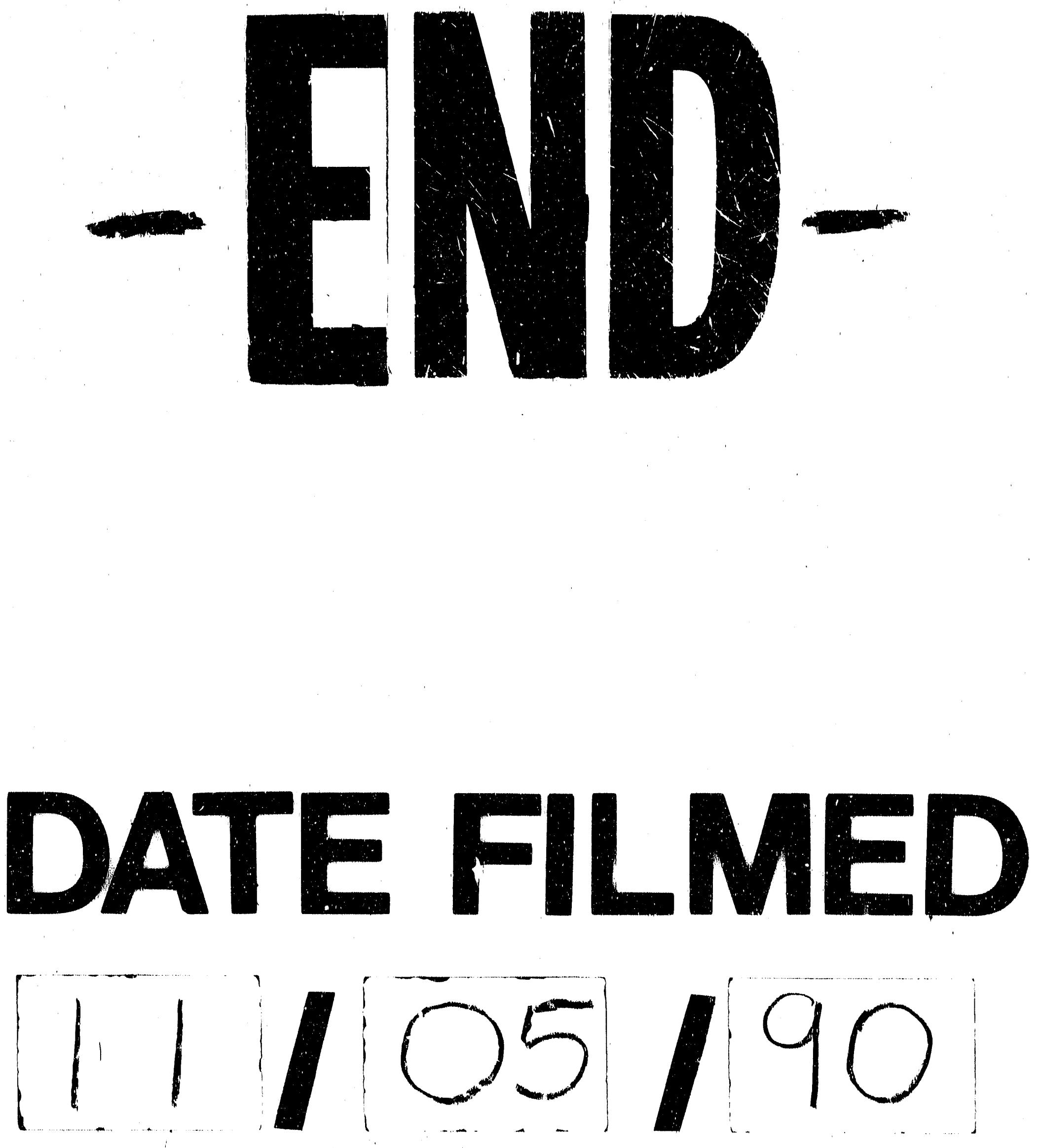
